Теорія Ймовір. та Матем. Статист. Вип. 74, 2006
Theor. Probability and Math. Statist.

No. 74, 2007, Pages 125-132

S 0094-9000(07)00702-8

Article electronically published on July 5, 2007

\title{
THE STATIONARY MEASURE OF THE STOCHASTIC TRANSPORT PROCESS WITH REFLECTING BARRIERS IN A SEMI-MARKOV ENVIRONMENT
}

UDC 519.21

\author{
A. O. POGORUI
}

\begin{abstract}
The stationary distribution is studied for the process described by stochastic evolution differential equations with reflecting barriers in a semi-Markov environment.
\end{abstract}

The transport process in a Markov environment is described by the equation 1, 2]

$$
\frac{d v(t)}{d t}=C(\kappa(t), v(t))
$$

where $\kappa(t)$ is a semi-Markov process. Let

$$
G=X \cup Y, \quad X=\left\{x_{1}, x_{2}, \ldots, x_{n}\right\}, \quad Y=\left\{y_{1}, y_{2}, \ldots, y_{m}\right\}
$$

be the phase space of the semi-Markov process $\kappa(t)$, let $P=\left\{p_{\alpha \beta}, \alpha, \beta \in G\right\}$ be the matrix of transition probabilities of the embedded (into $\kappa(t)$ ) ergodic Markov chain $\kappa_{l}$, $l \in N$, that is, $p_{\alpha \beta}=\mathrm{P}\left\{\kappa_{l+1}=\alpha / \kappa_{l}=\beta\right\}$, and let $\tau_{\alpha}$ be the time spent by the chain $\kappa_{l}$ at a state $\alpha \in G$. We assume that $\tau_{\alpha}$ is a random variable with a general distribution function $F_{\alpha}(t)$. We also assume the following.

C1. The distribution $F_{\alpha}$ is absolute continuous with the density $f_{\alpha}(t)=d F_{\alpha}(t) / d t$ such that the first two moments

$$
m_{\alpha}=\int_{0}^{\infty} t f_{\alpha}(t) d t, \quad m_{\alpha}^{(2)}=\int_{0}^{\infty} t^{2} f_{\alpha}(t) d t
$$

are finite for all $\alpha \in G$.

Let $V_{0}, V_{1}$ and $a_{i}, b_{i} \in \mathbf{R}$ be such that $V_{0}<V_{1}$ and $a_{i}>0, b_{i}>0$ for $i=1, \ldots, n$. Assume that the function $C$ on the right hand side of equation (1) is such that

$$
\begin{aligned}
& \text { for } x_{i} \in X, i=1, \ldots, n, \quad C\left(x_{i}, v\right)= \begin{cases}-a_{i}, & V_{0}<v \leq V_{1}, \\
0, & v=V_{0},\end{cases} \\
& \text { for } y_{j} \in Y, j=1, \ldots, m, \quad C\left(y_{j}, v\right)= \begin{cases}b_{j}, & V_{0} \leq v<V_{1}, \\
0, & v=V_{1} .\end{cases}
\end{aligned}
$$

Functions $C$ of this type appear in problems of the efficiency of multiphase systems with bunkers [3, 4.

We introduce a three component process $\xi$ on the phase space $Z=[0, \infty) \times G \times\left[V_{0}, V_{1}\right]$ :

$$
\xi(t)=(\tau(t), \kappa(t), v(t)),
$$

2000 Mathematics Subject Classification. Primary 60K15; Secondary 90C40.

Key words and phrases. Semi-Markov evolution, infinitesimal operator, stationary distribution. 
where

$$
\tau(t)=t-\sup \{u \leq t: \kappa(t) \neq \kappa(u)\} .
$$

Our aim is to study the stationary measure of the process $\xi(t)$. Note that $\xi(t)$ is a Markov process [5, 6]. Its infinitesimal operator is given by [3, 7]

$$
A \varphi(\tau, \alpha, v)=\frac{\partial}{\partial \tau} \varphi(\tau, \alpha, v)+r_{\alpha}(\tau)[P \varphi(0, \alpha, v)-\varphi(\tau, \alpha, v)]+C(\alpha, v) \frac{\partial}{\partial v} \varphi(\tau, \alpha, v)
$$

and the boundary conditions are

$$
\varphi_{\tau}^{\prime}\left(\tau, x, V_{1}\right)=\varphi_{\tau}^{\prime}\left(\tau, y, V_{0}\right)=0, \quad x \in X, y \in Y,
$$

where $r_{\alpha}(\tau)=f_{\alpha}(\tau) /\left(1-F_{\alpha}(\tau)\right)$ and

$$
P \varphi(0, \alpha, v)=\sum_{\beta \in G} p_{\alpha \beta} \varphi(0, \beta, v) .
$$

If the stationary distribution $\rho(\cdot)$ exists for the process $\xi(t)$, then

$$
\int_{Z} A \varphi(d z) \rho(d z)=0
$$

for all functions $\varphi(\cdot)$ of the domain of the operator $A$. It follows from the properties of the process $\xi(t)$ that the points $\left(\tau, x, V_{0}\right), x \in X$, and $\left(\tau, y, V_{1}\right), y \in Y$, of the phase space $Z$ are the atoms of the stationary measure $\rho(\cdot)$. In what follows, we denote these atoms by $\rho\left[\tau, x, V_{0}\right]$ and $\rho\left[\tau, y, V_{1}\right]$, respectively. The density of the measure is denoted by $\rho(\tau, x, v)$. Changing the order of integration in the integral on the left hand side of (2), we obtain $A^{*} \rho=0$ where $A^{*}$ is the conjugate operator, namely

$$
\begin{aligned}
\int_{Z} A \varphi(z) \rho(d z) & \\
=\sum_{\alpha \in G} & {\left[\int_{0}^{\infty} \int_{V_{0}}^{V_{1}} \rho(\tau, \alpha, v) \frac{\partial}{\partial \tau} \varphi(\tau, \alpha, v) d v d \tau+\int_{0}^{\infty} \int_{V_{0}}^{V_{1}} r_{\alpha}(\tau) \rho(\tau, \alpha, v) P \varphi(0, \alpha, v)\right.} \\
& \left.-\int_{0}^{\infty} \int_{V_{0}}^{V_{1}} r_{\alpha}(\tau) \rho(\tau, \alpha, v) \varphi(\tau, \alpha, v)+\int_{0}^{\infty} \int_{V_{0}}^{V_{1}} \rho(\tau, \alpha, v) C(\alpha, v) \frac{\partial}{\partial v} \varphi(\tau, \alpha, v)\right] \\
+ & \sum_{x \in X}\left[\int_{0}^{\infty} \rho\left[\tau, \alpha, V_{0}\right] \frac{\partial}{\partial \tau} \varphi\left(\tau, x, V_{0}\right) d \tau+\int_{0}^{\infty} r_{x}(\tau) \rho\left[\tau, x, V_{0}\right] P \varphi\left(0, x, V_{0}\right)\right. \\
\times & \sum_{y \in Y}\left[\int_{0}^{\infty} \rho\left[\tau, y, V_{1}\right] \frac{\partial}{\partial \tau} \varphi\left(\tau, y, V_{1}\right) d \tau+\int_{0}^{\infty} r_{y}(\tau) \rho\left[\tau, y, V_{1}\right] P \varphi\left(0, y, V_{1}\right)\right. \\
=\sum_{\alpha \in G} & {\left[\int_{V_{0}}^{V_{1}}\{\varphi(\infty, \alpha, v) \rho(\infty, \alpha, v)-\varphi(0, \alpha, v) \rho(0, \alpha, v)\right.} \\
& +\int_{V_{0}}^{V_{V_{1}}} \int_{0}^{\infty} r_{\alpha}(\tau) \rho(\tau, \alpha, v) d \tau P \varphi(0, \alpha, v) d v \\
& \left.\left.\quad \int_{0}^{\infty} \int_{V_{0}}^{V_{1}} \varphi(\infty, \alpha, v) r_{\alpha}(\tau) \rho(\tau, \alpha, v) d v d \tau, y, V_{1}\right] \varphi\left(\tau, y, V_{1}\right)\right] \\
& \left.-\int_{0}^{\infty} \varphi(\tau, \alpha, v) \frac{\partial}{\partial \tau} \rho(\tau, \alpha, v)\right\} d v
\end{aligned}
$$




$$
\begin{gathered}
+\int_{0}^{\infty}\left\{\varphi\left(\tau, \alpha, V_{1}\right) C\left(\alpha, V_{1}\right) \rho\left(\tau, \alpha, V_{1}-\right)\right. \\
\left.-\varphi\left(\tau, \alpha, V_{0}\right) C\left(\alpha, V_{0}\right) \rho\left(\tau, \alpha, V_{0}+\right)\right\} d \tau \\
\left.\quad+\int_{0}^{\infty} \int_{V_{0}}^{V_{1}} \varphi(\tau, \alpha, v) C(\alpha, v) \frac{\partial}{\partial v} \rho(\tau, \alpha, v) d v d \tau\right] \\
+\sum_{x \in X}\left[\rho\left[\infty, x, V_{0}\right] \varphi\left(\infty, x, V_{0}\right)-\rho\left[0, x, V_{0}\right] \varphi\left(0, x, V_{0}\right)\right. \\
-\int_{0}^{\infty} \varphi\left(\tau, x, V_{0}\right) \frac{\partial}{\partial \tau} \rho\left[\tau, x, V_{0}\right] d \tau+\int_{0}^{\infty} r_{x}(\tau) \rho\left[\tau, x, V_{0}\right] P \varphi\left(0, x, V_{0}\right) d \tau \\
\left.\quad-\int_{0}^{\infty} \varphi\left(\tau, x, V_{0}\right) r_{x}(\tau) \rho\left[\tau, x, V_{0}\right] d \tau\right] \\
+\sum_{y \in Y}\left[\rho\left[\infty, y, V_{1}\right] \varphi\left(\infty, y, V_{1}\right)-\rho\left[0, y, V_{1}\right] \varphi\left(0, y, V_{1}\right)\right. \\
-\int_{0}^{\infty} \varphi\left(\tau, y, V_{1}\right) \frac{\partial}{\partial \tau} \rho\left[\tau, y, V_{1}\right] d \tau+\int_{0}^{\infty} r_{y}(\tau) \rho\left[\tau, y, V_{1}\right] P \varphi\left(0, y, V_{1}\right) d \tau \\
\left.-\int_{0}^{\infty} \varphi\left(\tau, y, V_{1}\right) r_{y}(\tau) \rho\left[\tau, y, V_{1}\right] d \tau\right]
\end{gathered}
$$

$=0$.

Taking into account equality (2), we get the differential equations

$$
C(\alpha, v) \frac{\partial}{\partial v} \rho(\tau, \alpha, v)+r_{\alpha}(\tau) \rho(\tau, \alpha, v)+\frac{\partial}{\partial \tau} \rho(\tau, \alpha, v)=0
$$

integral equations

$$
\sum_{\beta \in G} \int_{0}^{\infty} r_{\beta}(\tau) \rho(\tau, \beta, v) d \tau p_{\beta \alpha}=\rho(0, \beta, v), \quad \alpha \in G
$$

and the boundary conditions $\rho(\infty, \alpha, v)=0, \alpha \in G$, for the densities as well as the differential equations

$$
\begin{gathered}
\frac{d}{d \tau} \rho\left[\tau, x, V_{0}\right]+r_{x}(\tau) \rho\left[\tau, x, V_{0}\right]-a_{x} \rho\left(\tau, x, V_{0}+\right)=0, \\
\frac{d}{d \tau} \rho\left[\tau, y, V_{1}\right]+r_{y}(\tau) \rho\left[\tau, y, V_{1}\right]-b_{y} \rho\left(\tau, y, V_{1}-\right)=0
\end{gathered}
$$

for the atoms of the distribution where

$$
\rho\left(\tau, x, V_{0}+\right)=\lim _{v \downarrow V_{0}} \rho(\tau, x, v), \quad \rho\left(\tau, y, V_{1}\right)=\lim _{v \uparrow V_{1}} \rho(\tau, y, v) .
$$

The atoms of the measure satisfy the integral equations

$$
\begin{gathered}
\sum_{x \in X} \int_{0}^{\infty} r_{x}(\tau) \rho\left[\tau, x, V_{0}\right] d \tau p_{x z}=\rho\left[0, z, V_{0}\right], \quad z \in X, \\
\sum_{y \in Y} \int_{0}^{\infty} r_{y}(\tau) \rho\left[\tau, y, V_{1}\right] d \tau p_{y z}=\rho\left[0, z, V_{1}\right], \quad z \in Y, \\
\sum_{x \in X} \int_{0}^{\infty} r_{x}(\tau) \rho\left[\tau, x, V_{0}\right] d \tau p_{x y}=b_{y} \int_{0}^{\infty} \rho\left(\tau, y, V_{0}+\right), \quad y \in Y, \\
\sum_{y \in Y} \int_{0}^{\infty} r_{y}(\tau) \rho\left[\tau, y, V_{1}\right] d \tau p_{y x}=a_{x} \int_{0}^{\infty} \rho\left(\tau, x, V_{1}-\right) d \tau, \quad x \in X,
\end{gathered}
$$


and the boundary conditions

$$
\rho\left[\infty, x, V_{0}\right]=\rho\left[\infty, y, V_{1}\right]=0, \quad x \in X, y \in Y .
$$

Solving equations (3) and taking into account that

$$
\exp \left\{-\int_{0}^{\tau} r_{\alpha}(s) d s\right\}=1-F_{\alpha}(\tau),
$$

we get

$$
\begin{array}{cc}
\rho(\tau, x, v)=h_{x}\left(v+a_{x} \tau\right)\left(1-F_{x}(\tau)\right), & x \in X, \\
\rho(\tau, y, v)=h_{y}\left(v-b_{y} \tau\right)\left(1-F_{y}(\tau)\right), & y \in Y .
\end{array}
$$

Considering equation (4), we seek for a function $h_{\alpha}(\cdot), \alpha \in G$, of the form

$$
h_{\alpha}(x)=c_{\alpha} e^{s x}, \quad c_{\alpha}>0, \alpha \in G .
$$

Then equation (4) implies the system of equations

$$
\sum_{\beta \in G} c_{\beta} \int_{0}^{\infty} f_{\beta}(\tau) e^{s k_{\beta} \tau} d \tau p_{\beta \alpha}=c_{\alpha}, \quad \alpha \in G,
$$

where

$$
k_{\beta}= \begin{cases}a_{\beta}, & \beta \in X, \\ -b_{\beta}, & \beta \in Y .\end{cases}
$$

The Laplace transform of the function $f_{\beta}(\tau)$ is given by

$$
\widehat{f}_{\beta}\left(s k_{\beta}\right)=\int_{0}^{\infty} f_{\beta}(\tau) e^{s k_{\beta} \tau} d \tau .
$$

It is obvious that the system (10) with respect to unknowns $c_{\alpha}, \alpha \in G$, has a solution if the determinant of the system is zero, that is,

$$
\left|\begin{array}{cccc}
-1 & \widehat{f}_{x_{1}}\left(a_{1} s\right) p_{x_{1} x_{2}} & \ldots & \widehat{f}_{x_{1}}\left(a_{1} s\right) p_{x_{1} y_{m}} \\
\widehat{f}_{x_{2}}\left(a_{2} s\right) p_{x_{2} x_{1}} & -1 & \ldots & \widehat{f}_{x_{2}}\left(a_{2} s\right) p_{x_{2} y_{m}} \\
- & - & - & - \\
\widehat{f}_{y_{m}}\left(-b_{m} s\right) p_{y_{m} x_{1}} & \widehat{f}_{y_{m}}\left(-b_{m} s\right) p_{y_{m} x_{2}} & \ldots & -1
\end{array}\right|=0 .
$$

The case where the balance condition holds. It is easy to check that $s=0$ is a solution of (11). If the solution is unique, then equation (10) becomes of the form

$$
\sum_{\beta \in G} c_{\beta} p_{\beta \alpha}=c_{\alpha}, \quad \alpha \in G .
$$

This implies that $c_{\alpha}=\sigma \rho_{\alpha}$ where $\rho_{\alpha}, \alpha \in G$, is the stationary distribution of the embedded Markov chain $\kappa_{l}, l \in \mathbf{N}$, and $\sigma$ is the normalizing factor defined from the condition $\int_{Z} \rho(z) d z=1$. Then $h_{\alpha}(x)=\sigma \rho_{\alpha}$ and (9) yields

$$
\rho(\tau, x, v)=\sigma \rho_{x}\left(1-F_{x}(\tau)\right), \quad x \in X, \quad \rho(\tau, y, v)=\sigma \rho_{y}\left(1-F_{y}(\tau)\right), \quad y \in Y .
$$

Solving equation (5) and taking into account (12), we get

$$
\begin{aligned}
& \rho\left[\tau, x, V_{0}\right]=\sigma \rho_{x}\left(1-F_{x}(\tau)\right)\left(a_{x} \tau+\frac{\rho\left[0, x, V_{0}\right]}{\sigma \rho_{x}}\right), \\
& \rho\left[\tau, y, V_{1}\right]=\sigma \rho_{y}\left(1-F_{y}(\tau)\right)\left(b_{y} \tau+\frac{\rho\left[0, y, V_{1}\right]}{\sigma \rho_{y}}\right) .
\end{aligned}
$$


Substituting these results in (7), we obtain

$$
\begin{aligned}
& \sigma \sum_{x \in X} \rho_{x} a_{x} m_{x} p_{x z}+\sum_{x \in X} \rho\left[0, x, V_{0}\right] p_{x z}=\rho\left[0, z, V_{0}\right], \quad z \in X, \\
& \sigma \sum_{y \in Y} \rho_{y} b_{y} m_{y} p_{y z}+\sum_{y \in Y} \rho\left[0, y, V_{1}\right] p_{y z}=\rho\left[0, z, V_{1}\right], \quad z \in Y, \\
& \sigma \sum_{y \in Y} \rho_{y} b_{y} m_{y} p_{y x}+\sum_{y \in Y} \rho\left[0, y, V_{1}\right] p_{y x}=\sigma \rho_{x} a_{x} m_{x}, \quad x \in X, \\
& \sigma \sum_{x \in X} \rho_{x} a_{x} m_{x} p_{x y}+\sum_{x \in X} \rho\left[0, x, V_{0}\right] p_{x y}=\sigma \rho_{y} b_{y} m_{y}, \quad y \in Y .
\end{aligned}
$$

Put $P_{X}=\left\{p_{x z}, x, z \in X\right\}, P_{Y}=\left\{p_{x z}, x, z \in Y\right\}$, and

$$
G_{X}=\left(I-P_{X}\right)^{-1}=\left\{g_{x z}, x, z \in X\right\}, \quad G_{Y}=\left(I-P_{Y}\right)^{-1}=\left\{g_{y z}, y, z \in Y\right\} .
$$

Since the Markov chain $\kappa_{l}$ is ergodic, the matrices $G_{X}$ and $G_{Y}$ exist and are potentials [6]. Solving the first two equations of (14), we have

$$
\begin{aligned}
& \rho\left[0, z, V_{0}\right]=\sigma \sum_{x \in X} a_{x} m_{x} \rho_{x} \sum_{k \in X} p_{x k} g_{k z}, \quad z \in X, \\
& \rho\left[0, z, V_{1}\right]=\sigma \sum_{y \in Y} b_{y} m_{y} \rho_{y} \sum_{k \in Y} p_{y k} g_{k z}, \quad z \in Y .
\end{aligned}
$$

Substituting these relations in equality (14), we obtain the condition for the existence of the stationary distribution of the process (the so-called balance condition):

C2. We have

$$
\begin{array}{ll}
\sum_{x \in X} \rho_{x} a_{x} m_{x} \sum_{z \in X} g_{x z} p_{z y}=\rho_{y} b_{y} m_{y}, & y \in Y, \\
\sum_{y \in Y} \rho_{y} a_{y} m_{y} \sum_{z \in Y} g_{y z} p_{z x}=\rho_{x} b_{x} m_{x}, & x \in X .
\end{array}
$$

Therefore we proved the following result.

Theorem 1. If conditions $C 1$ and C2 hold, then the stationary distribution $\rho(\cdot)$ of the process $\xi(t)$ is characterized by the equalities

$$
\rho(\tau, x, v)=\sigma \rho_{x}\left(1-F_{x}(\tau)\right), \quad x \in X, \quad \rho(\tau, y, v)=\sigma \rho_{y}\left(1-F_{y}(\tau)\right), \quad y \in Y,
$$

for densities and

$$
\begin{gathered}
\rho\left[\tau, x, V_{0}\right]=\sigma \rho_{x}\left(1-F_{x}(\tau)\right)\left(a_{x} \tau+\frac{\sum_{z \in X} a_{z} m_{z} \rho_{z} \sum_{k \in X} p_{z k} g_{k x}}{\rho_{x}}\right), \\
\rho\left[\tau, y, V_{1}\right]=\sigma \rho_{y}\left(1-F_{y}(\tau)\right)\left(b_{y} \tau+\frac{\sum_{z \in Y} b_{z} m_{z} \rho_{z} \sum_{k \in Y} p_{z k} g_{k y}}{\rho_{y}}\right)
\end{gathered}
$$

for atoms.

The case where the balance does not hold. Now we consider the case where the solution of equation (11) is not unique. Denote by $\left\{s_{0}=0, s_{1}, \ldots, s_{l}\right\}, l \geq 1$, the set of solutions of equation (11). Every $s_{i}$ of this set corresponds to a solution $\left\{c_{\alpha}^{i}, \alpha \in G\right\}$ of equation (10) being unique up to a constant factor $\left(s_{0}=0\right.$ corresponds 
to the solution $c \rho_{\alpha}$ ). Thus we seek densities of the form

$$
\begin{array}{ll}
\rho(\tau, x, v)=\sum_{i=0}^{l} c_{x}^{i} e^{s_{i}\left(v+a_{x} \tau\right)}\left(1-F_{x}(\tau)\right), & x \in X, \\
\rho(\tau, y, v)=\sum_{i=0}^{l} c_{y}^{i} e^{s_{i}\left(v-b_{y} \tau\right)}\left(1-F_{y}(\tau)\right), & y \in Y .
\end{array}
$$

Solving equation (5) and taking into account (15), we have

$$
\begin{aligned}
\rho\left[\tau, x, V_{0}\right]= & \sum_{i=1}^{l} \frac{c_{x}^{i}}{s_{i}}\left(1-F_{x}(\tau)\right) e^{s_{i} V_{0}}\left(e^{a_{x} s_{i} \tau}-1\right)+c \rho_{x} a_{x} \tau\left(1-F_{x}(\tau)\right) \\
& +\rho\left[0, x, V_{0}\right]\left(1-F_{x}(\tau)\right), \quad x \in X, \\
\rho\left[\tau, y, V_{1}\right]= & \sum_{i=1}^{l} \frac{c_{y}^{i}}{s_{i}}\left(1-F_{y}(\tau)\right) e^{s_{i} V_{1}}\left(1-e^{-b_{y} s_{i} \tau}\right)+c \rho_{y} b_{y} \tau\left(1-F_{y}(\tau)\right) \\
& +\rho\left[0, y, V_{1}\right]\left(1-F_{y}(\tau)\right), \quad y \in Y .
\end{aligned}
$$

Substituting (16) in the first two equations of ([6), we get

$$
\begin{aligned}
\rho\left[0, z, V_{0}\right]= & \sum_{i=1}^{l} \frac{e^{s_{i} V_{0}}}{s_{i}} \sum_{x \in X}\left[c_{x}^{i}\left(\widehat{f}\left(s_{i} a_{x}\right)-1\right) \sum_{k \in X} p_{x k} g_{k z}\right] \\
& +c \sum_{x \in X} \rho_{x} a_{x} m_{x} \sum_{k \in X} p_{x k} g_{k z}, \quad z \in X, \\
\rho\left[0, z, V_{1}\right]= & \sum_{i=1}^{l} \frac{e^{s_{i} V_{1}}}{s_{i}} \sum_{y \in Y}\left[c_{y}^{i}\left(1-\widehat{f}\left(-s_{i} b_{y}\right)\right) \sum_{k \in Y} p_{y k} g_{k z}\right] \\
& +c \sum_{y \in Y} \rho_{y} b_{y} m_{y} \sum_{k \in Y} p_{y k} g_{k z}, \quad z \in Y .
\end{aligned}
$$

To find $\left\{c_{\alpha}^{i}, \alpha \in G\right\}$, we combine these equalities and the last two equations in ([6). This leads to the following condition.

C3. Among solutions $\left\{c_{\alpha}^{i}, \alpha \in G\right\}$ of equations (10) that correspond to $s_{i}$, there are nonzero solutions $\left\{c_{\alpha}^{i_{r}}, \alpha \in G, r=1, \ldots, d\right\}, r \leq l$, such that

$$
\begin{gathered}
\sum_{r=1}^{d} \sum_{x \in X}\left[c_{x}^{i_{r}}\left(\widehat{f}\left(s_{i_{r}} a_{x}\right)-1\right) \sum_{z \in X} g_{x z} p_{z y}\right]+c \sum_{x \in X} \rho_{x} a_{x} m_{x} \sum_{z \in X} g_{x z} p_{z y} \\
=\sum_{r=1}^{d} c_{x}^{i_{r}}\left(1-\widehat{f}\left(-s_{i_{r}} b_{y}\right)\right)+c \rho_{y} b_{y} m_{y}, \quad y \in Y, \\
\sum_{r=1}^{d} \sum_{y \in Y}\left[c_{y}^{i_{r}}\left(1-\widehat{f}\left(-s_{i_{r}} b_{y}\right)\right) \sum_{z \in Y} g_{y z} p_{z x}\right]+c \sum_{y \in Y} \rho_{y} a_{y} m_{y} \sum_{z \in Y} g_{y z} p_{z x} \\
=\sum_{r=1}^{d} c_{x}^{i_{r}}\left(\widehat{f}\left(s_{i_{r}} a_{x}\right)-1\right)+c \rho_{x} a_{x} m_{x}, \quad x \in X .
\end{gathered}
$$

Therefore we have proved the following result. 
Theorem 2. If conditions $C 1$ and $C 3$ hold, then the Markov process $\xi(t)$ has the stationary distribution $\rho(\cdot)$ whose density is given by

$$
\begin{array}{ll}
\rho(\tau, x, v)=\sigma_{1} \sum_{r=1}^{d} c_{x}^{i_{r}} e^{s_{i_{r}}\left(v+a_{x} \tau\right)}\left(1-F_{x}(\tau)\right), & x \in X, \\
\rho(\tau, y, v)=\sigma_{1} \sum_{r=1}^{d} c_{y}^{i_{r}} e^{s_{i_{r}}\left(v-b_{y} \tau\right)}\left(1-F_{y}(\tau)\right), & y \in Y,
\end{array}
$$

where $c_{\alpha}^{o}=c \rho_{\alpha}$, and whose atoms are given by

$$
\begin{aligned}
\rho\left[\tau, x, V_{0}\right]=\sigma_{1}\left[\sum_{i=1}^{l} \frac{c_{x}^{i}}{s_{i}}\left(1-F_{x}(\tau)\right) e^{s_{i} V_{0}}\left(e^{a_{x} s_{i} \tau}-1\right)\right. & +c \rho_{x} a_{x} \tau\left(1-F_{x}(\tau)\right) \\
& \left.+\rho\left[0, x, V_{0}\right]\left(1-F_{x}(\tau)\right)\right], \quad x \in X, \\
\rho\left[\tau, y, V_{1}\right]=\sigma_{1}\left[\sum_{i=1}^{l} \frac{c_{y}^{i}}{s_{i}}\left(1-F_{y}(\tau)\right) e^{s_{i} V_{1}}\left(1-e^{-b_{y} s_{i} \tau}\right)+c \rho_{y} b_{y} \tau\left(1-F_{y}(\tau)\right)\right. & \\
\left.+\rho\left[0, y, V_{1}\right]\left(1-F_{y}(\tau)\right)\right], \quad y \in Y, &
\end{aligned}
$$

where $\sigma_{1}$ is defined from the condition $\int_{Z} \rho(z) d z=1$.

Note also that the case of $c=0$ is not excluded.

Example. Consider Theorem 2 for the case of $n=m=1$. Then $X=\{x\}, Y=\{y\}$, the matrix of transient probabilities $P$ of the embedded (to the process $\kappa(t)$ ) Markov chain $\kappa_{l}$ is $P=\left(\begin{array}{ll}1 & 0 \\ 0 & 1\end{array}\right)$, and equation (11) is

$$
\widehat{f}_{x}(s a) \widehat{f}_{y}(-s b)=1 .
$$

Assume that the balance condition does not hold. Let $s_{i}$ satisfy (18). Then the corresponding $\left\{c_{x}^{i}, c_{y}^{i}\right\}$ satisfy equalities

$$
c_{x}^{i} \widehat{f}_{x}\left(s_{i} a\right)=c_{y}^{i}, \quad c_{y}^{i} \widehat{f}_{y}\left(-s_{i} b\right)=c_{x}^{i}, \quad i=1, \ldots, l,
$$

and condition $\mathrm{C} 3$ becomes of the form

$$
\sum_{i=1}^{l} c_{x}^{i}\left(\widehat{f}_{x}(s a)-1\right)+c a_{x} m_{x} \rho_{x}=\sum_{i=1}^{l} c_{y}^{i}\left(1-\widehat{f}_{y}(-s b)\right)+c a_{y} m_{y} \rho_{y} .
$$

Since the balance condition does not hold, the latter condition holds for $c=0$ only. Thus the density of the stationary distribution is given by

$$
\rho(\tau, x, v)=\sigma_{1} \sum_{i=1}^{l} c_{x}^{i} e^{s_{i}(v+a \tau)}\left(1-F_{x}(\tau)\right), \quad \rho(\tau, y, v)=\sigma_{1} \sum_{i=1}^{l} c_{y}^{i} e^{s_{i}(v-b \tau)}\left(1-F_{x}(\tau)\right),
$$

while the atoms are given by

$$
\begin{gathered}
\rho\left[0, x, V_{0}\right]=\sigma_{1} \sum_{i=1}^{l} \frac{e^{s_{i} V_{0}}}{s_{i}} c_{x}^{i} \widehat{f}\left(s_{i} a\right), \\
\rho\left[0, y, V_{1}\right]=\sigma_{1} \sum_{i=1}^{l} \frac{e^{s_{i} V_{1}}}{s_{i}} c_{y}^{i} \widehat{f}\left(-s_{i} b\right) .
\end{gathered}
$$


In order to apply the above result, one needs to know the stationary distribution $\bar{\rho}(\cdot)$ of the process $\zeta(\kappa(t), v(t))[3$, 4]. This distribution can be found from the distribution of $\rho(\cdot)$ by

$$
\bar{\rho}(\cdot)=\int_{0}^{\infty} \rho(\tau, \cdot) d \tau .
$$

Let $f_{0}(x)=\lambda e^{-\lambda x}$ and $f_{1}(x)=p^{2} x e^{-p x}$. Then

$$
\widehat{f}_{o}(s a) \widehat{f}_{1}(-s b)=\frac{\lambda p^{2}}{(\lambda-a s)(p+b s)^{2}}=1 .
$$

The integral $\int_{0}^{\infty} \rho(\tau, \cdot) d \tau$ converges if $a s_{i}\left\langle\lambda\right.$ and $b s_{i}>-p$. These assumptions also imply that equation (19) has the unique solution

$$
s_{1}=\frac{b \lambda-2 a p+\sqrt{4 a b p \lambda+(b \lambda)^{2}}}{2 a b} .
$$

This result allows us to find the stationary distribution of $\rho(\cdot)$. The balance condition holds in this case if $2 b \lambda=a p$.

Remark. We assume in Theorem 2 that the roots of equation (11) are real and the number of roots is finite. If we omit this assumption and assume instead that the stationary distribution is a convergent Fourier series, then the result may have more applications. However this case requires a deeper consideration that will be published elsewhere.

\section{BIBLIOGRAPHY}

1. V. S. Korolyuk and V. V. Korolyuk, Stochastic Models of Systems, Lybid', Kyiv, 1993; English transl., Kluwer Academic Publishers, Dordrecht, 1999. MR.1817881 MR1753470(2002b:60169)

2. V. S. Korolyuk and A. V. Svishchuk, Semi-Markov Random Evolutions, Lybid', Kyiv, 1992; English transl., Kluwer Academic Publishers, Dordrecht, 1995. MR1286045 (95j:60143) MR.1472977 (98e:60145)

3. A. A. Pogorul̆ and A. F. Turbin, Estimation of stationary efficiency of a production line with two unreliable aggregates, Kibernetika i Sistemnyi Analiz 6 (2002), 35-42; English transl. in Cybernetics and Systems Analysis 38 (2002), no. 6, 823-829.

4. A. F. Turbin and A. A. Pogoruĭ, Computation of stationary efficiency indices for inventory control systems with feedback, Intellectualization of Systems for Processing Informational Messages, Nats. Akad. Nauk Ukrainy, Inst. Mat., Kyiv, 1995, pp. 191-204. (Russian) MR 1365762

5. I. I. Gikhman and A. V. Skorokhod, The Theory of Stochastic Processes, vol. II, Nauka, Moscow, 1973; English transl., Springer-Verlag, New York-Heidelberg, 1975. MR2058260 (2005a:60003)

6. V. S. Korolyuk and A. F. Turbin, Mathematical Foundations of the State Lumping of Large Systems, Kluwer Academic Publishers, Dordrecht, 1994. MR.1281385 (95e:60071)

7. A. N. Korlat, V. N. Kuznetsov, M. M. Novikov, and A. F. Turbin, Semi-Markov Repairable Systems and Queuing Systems, Shtiintsa, Kishinev, 1991. (Russian) MR.1187752 (94e:60075)

Department of Mathematics, Zhytomyr Pedagogical University, Velyka Berdychevs'ka Street, 40, 10008 Zhytomyr, Ukraine

E-mail address: pogor@zspu.edu.ua

Received 23/DEC/2003

Translated by OLEG KLESOV 